\title{
X-linked parkinsonism-spasticity syndrome
}

INSERM

\section{Source}

INSERM. (1999). Orphanet: an online rare disease and orphan drug data base. $\underline{X-l i n k e d}$ parkinsonism-spasticity syndrome. ORPHA:363654

X-linked parkinsonism-spasticity syndrome is a rare genetic neurological disorder characterized by parkinsonian features (including resting or action tremor, cogwheel rigidity, hypomimia and bradykinesia) associated with variably penetrant spasticity, hyperactive deep tendon reflexes and Babinski sign. 